\title{
High efficiency operation of butt joint line-defect-waveguide microlaser in two-dimensional photonic crystal slab
}

\author{
Wanhua Zheng, ${ }^{1, a)}$ Gang Ren, ${ }^{1}$ Mingxin Xing, ${ }^{1}$ Wei Chen, ${ }^{1}$ Anjin Liu, ${ }^{1}$ Wenjun Zhou, ${ }^{1}$ \\ Toshihiko Baba, ${ }^{2}$ Kango Nozaki, ${ }^{2}$ and Lianghui Chen ${ }^{1}$ \\ ${ }^{1}$ Nano-Optoelectronics Laboratory, Institute of Semiconductors, CAS, Beijing 100083, People's Republic of \\ China \\ ${ }^{2}$ Department of Electrical and Computer Engineering, Yokohama National University, 79-5 Tokiwadai, \\ Hodogayaku, Yokohama 240-8501, Japan
}

(Received 6 March 2008; accepted 17 July 2008; published online 26 August 2008)

\begin{abstract}
Butt joint line-defect-waveguide microlasers are demonstrated on photonic crystal slabs with airholes in a triangular lattice. Such microlaser is designed to increase the output power from the waveguide edge directly. The output power is remarkably enhanced to 214 times higher by introducing chirped structure in the output waveguide. The lasing mode operates in the linear dispersion region of the output waveguide so that the absorption loss due to the band-edge effect is reduced. The laser resonance is illustrated theoretically using the finite difference time domain method. A practical high power efficiency of $20 \%$ is obtained in this microlaser. (C) 2008 American Institute of Physics. [DOI: 10.1063/1.2973207]
\end{abstract}

Photonic crystal (PC) is acknowledged as an enabling science and technology of the manipulating photons in the size of micrometers. The research in PC integrated circuits is driven by the advantages seen in the variety of twodimensional (2D) PC slab devices, such as the ultrasmall cavity laser, ultralow loss sharp bend waveguide, high performance and small sized beam splitters, and so on. Analogous to electronic integrated circuits, integrating photonic functions on a PC chip allow the realization of a cheaper and more compact optical systems with higher performance. As one of the key components, PC edge-emitting microcavity laser attracts many interests and their lasing performances have been experimentally demonstrated in two categories by some groups. One category is the line-defect-waveguide (LDW) laser. ${ }^{1-3}$ The resonant mode in LDW possesses an extremely high quality factor $\left(Q_{\|}\right.$and $\left.Q_{\perp}\right)$ in parallel and vertical directions due to the laser operation at the bandedge, and that leads to the extremely low laser output from the edge, which is proportional to $1 / Q_{\|}{ }^{3,6}$ The other one is the cavity-waveguide coupling system. ${ }^{410}$ Such system consists of a separated point defect microcavity and an output coupled waveguide in which the coupling efficiency between cavity and waveguide is the main issue. By increasing the overlap between the evanescent cavity field and the waveguide mode, up to $80 \%$ coupling efficiency is obtained in the H0 cavity-waveguide system, ${ }^{5}$ and $90 \%$ in L3 cavitywaveguide system, ${ }^{6}$ respectively. In this letter, we proposed a system which consists of one typical LDW butt jointed with one chirped LDW. Such system takes the advantage of a higher output power for a larger resonant area in the LDW and an intrinsic direct extraction of photons from the resonant mode to the output waveguide due to lower cavity $Q_{\|}$ factor in plane.

The platform of the device is a standard PC slab with airholes in a triangular lattice, which has a TE photonic bandgap. As shown in Fig. 1, the laser system is a simple line defect of missing airholes in $\Gamma-J$ direction, which consists of one LDW and one butt joint output waveguide. One end

${ }^{a)}$ Electronic mail: whzheng@red.semi.ac.cn. of the LDW is terminated by the PC, while the other end is butt jointed to an output waveguide. Such a cavity allows lasing without any laser mirrors due to zero group velocity at the photonic band edge. ${ }^{1}$ There are two key reasons for us to choose such butt jointed structure. One is to have an intrinsic mode coupling from the LDW laser resonant mode to the butt jointed output waveguide propogating mode. The resonant mode in the output waveguide region becomes a propagating mode intrinsically, which indicates a low coupling loss in this system. Another reason is the mechanism of the LDW laser. Such laser is based on the band-edge effect which will be expected to have a $2 \mathrm{D}$ coherent lasing over a large area and lead to a breakthrough in the limitation of point defect cavity. Therefore a higher power output could be expected. We believe that this is an important breakthrough

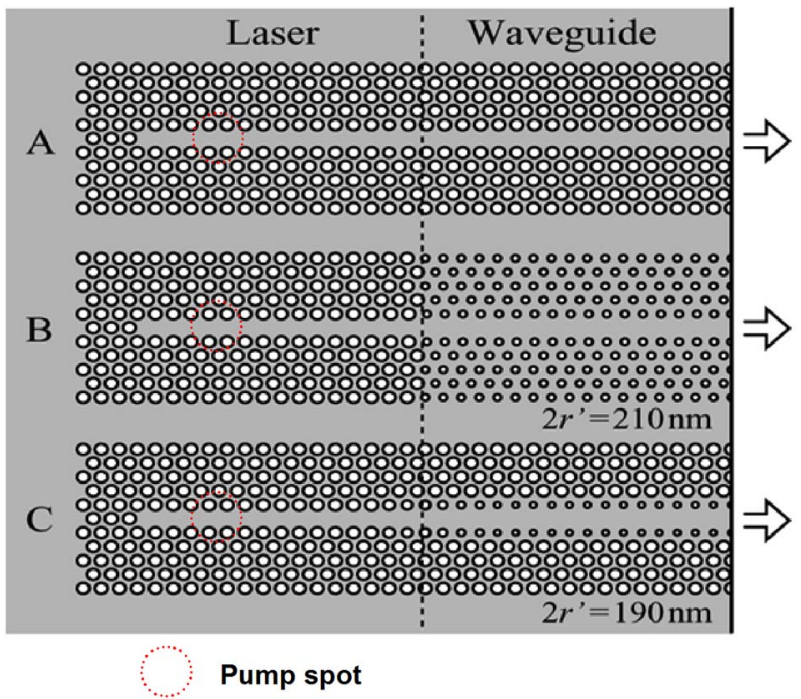

FIG. 1. (Color online) Schematic of the three laser systems. $2 r^{\prime}$ denotes diameter of smaller airholes. Waveguide $A$ has the same hole radius as the LDW cavity, waveguide $B$ has the smaller airholes of $210 \mathrm{~nm}$, and waveguide $C$ has the same structure as the LDW area except the airholes adjacent to the line defect. The diameter of the adjacent airholes is chirped from 260 to $190 \mathrm{~nm}$. The dotted red circles indicate the location of pump spots in the experiment. 


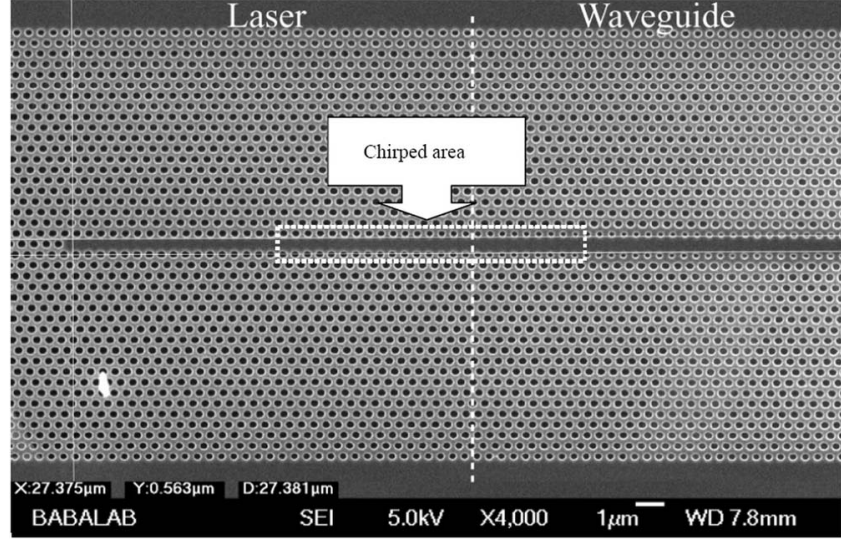

FIG. 2. Scanning electron microscopy image of the laser system $C$ of chirped waveguide butt joint LDW laser. The airholes enclosed by dashed line is the chirped area.

for the realization of a functional 2D optical chip.

In this work, the lattice constant in LDW laser was 420 $\mathrm{nm}$, and the airhole diameter $2 r$ of 2D PC area was $260 \mathrm{~nm}$. The LDW laser has 18 missing airholes along $\Gamma-J$ direction and one end is terminated using 20 periods of $2 \mathrm{D}$ PC in practical device. We prepared three types of output waveguides $A, B$, and $C$. Waveguide $A$ has the same structure as the LDW laser with 47 missing airholes. Therefore, the only difference between the laser and the output waveguide is whether they are optically excited or not in the experiment. Waveguide $B$ has a smaller filling factor of airholes $(210 \mathrm{~nm})$ but the same lattice constant in its whole structure. It pulls the target photonic band down to a lower frequency due to the effective refractive index increase. The laser resonance mode at the LDW band-edge frequency can operate in the linear dispersion region of the output waveguide to decrease the absorption loss due to the increasing group velocity. In addition, the resonance mode will be directly coupled to the waveguide mode for the perfect mode pattern matching in the butt joint structure. However, the suddenly airholes change at the interface between LDW and output waveguide will lead to strong diffraction and reflection losses. ${ }^{11}$ Waveguide $C$ has the same structure as the LDW area except the airholes adjacent to the line defect. The diameter of the adjacent airholes is chirped from 260 to $190 \mathrm{~nm}$ in 25 periods. This system is aimed to overcome the problem of sharp interface and is expected to have high performance.

In the experiment, we used a metal-organic chemicalvapor deposition-grown InGaAsP/InP wafer including seven $9 \mathrm{~nm}$ thick quantum wells (QWs) whose gain peak was at $1.538 \mu \mathrm{m}$. The device was fabricated by $e$-beam lithography, HI/Xe inductively coupled plasma etching of epilayers, ${ }^{12}$ and selective wet etching of InP layers, as shown in Fig. 2. The lasing was observed at room temperature by pulsed photopumping at $980 \mathrm{~nm}$ with a duty ratio of $0.075 \%$. The pump spot diameter was $\sim 3.5 \mu \mathrm{m}$, so the area overlapped with the line defect cavity was $38 \%$. The light output from the cleaved waveguide facet was detected from the side using a tipped multimode fiber and analyzed using a spectrometer. The near field pattern observed from the top is shown in Fig. 3. The light output from the pump position is so weak that no output signal is detected, which is coincident with the extremely high quality factor in the vertical direction for such band-edge mode. ${ }^{3}$ However, the light output

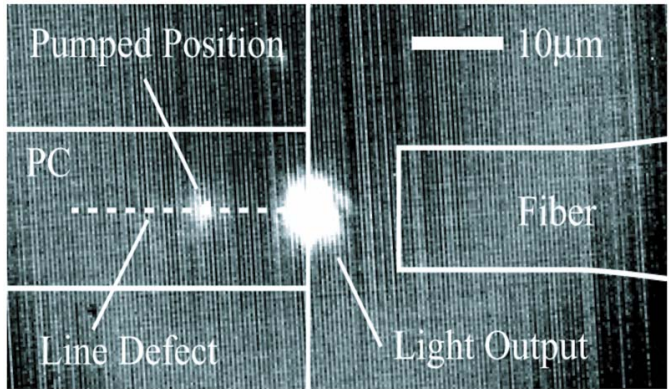

FIG. 3. (Color online) Near field pattern of laser excitation and output performance from the laser system $C$.

from the facet edge of the coupling waveguide is extremely strong. Furthermore, the light scattering from the output waveguide is undetected by the infrared charge-coupled device camera, which implies almost no vertical scattering loss in the devices. Among the three PC lasers, device $C$ illustrates the highest output power, and its lasing characteristics are shown in Fig. 4. The threshold pump power is $1.1 \mathrm{~mW}$. The absorption efficiency of the pump light is estimated to be $11 \%$ when the absorption coefficient of the pump light in the QWs is assumed to be $20000 \mathrm{~cm}^{-1}$. By considering this efficiency and the overlap efficiency of the pumped light with the line defect, the threshold can be converted to an effective pump power of $46 \mu \mathrm{W}$. The lasing spectrum shows a clear single mode operation with a large side mode suppression ratio of $37 \mathrm{~dB}$.

To illustrate the lasing performance, the cavity $Q$ factor and the electromagnetic-field distribution of the devices have been calculated using the $2 \mathrm{D}$ finite difference time domain

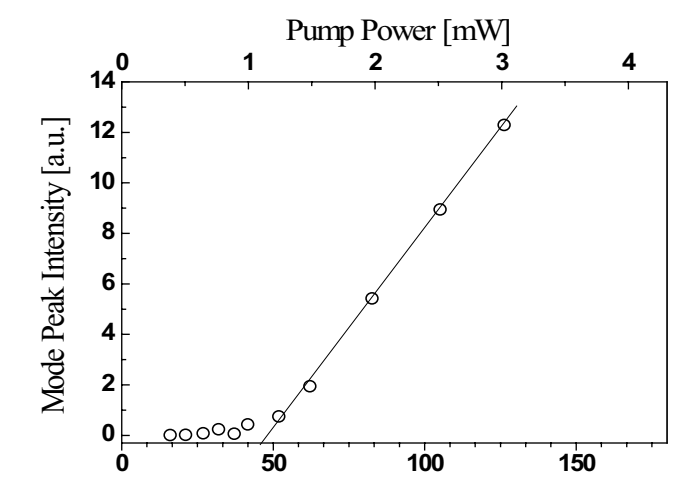

(a)

Effective Pump Power $[\mu \mathrm{W}]$

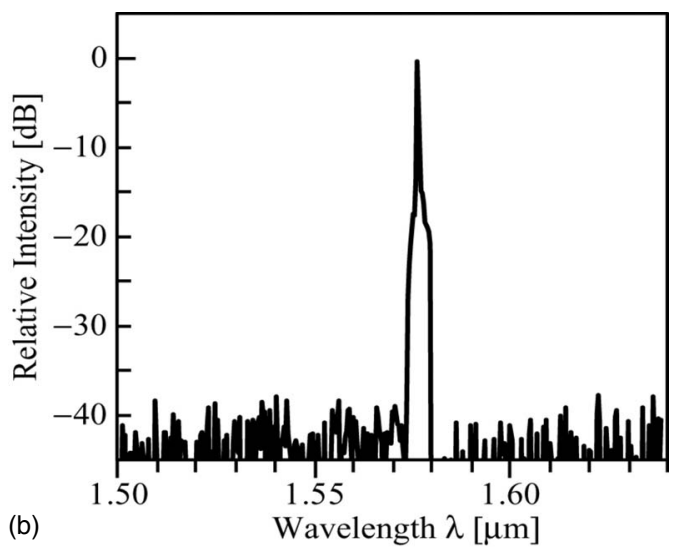

FIG. 4. (a) $L-L$ characteristic at peak power observed for the laser system $C$. (b) Lasing spectrum at peak power observed for the laser system $C$. 

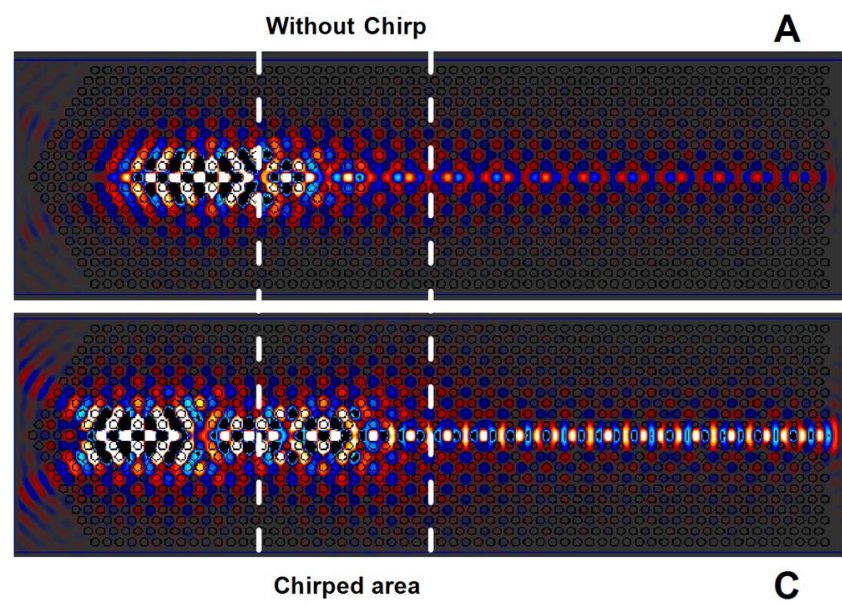

FIG. 5. (Color online) The vertical ( $y$ direction) magnetic-field distribution in the laser system $A$ (upper) and $C$ (lower).

(FDTD) method for the loss in the vertical direction could be ignored. An impulse light source is launched along $\Gamma-K$ direction to excite all the guided modes in this direction. A time monitor is located behind the light source. The mode with a slow-wave effect could be reflected using the Bragg scattering and thus be detected by the monitor. After the fast Fourier transform analysis, we can find the precise resonant frequency of the low group velocity modes on the transmission spectrum. The $Q$ factor is calculated by its definition:

$$
Q=\omega_{0} \frac{U(t)}{P(t)} .
$$

The structure is surrounded and covered by monitors so that the power loss $P(t)$ and the energy $U(t)$ in the whole structure can be calculated.

To demonstrate the laser emission from the edge of the LDW laser, Fig. 5 shows the vertical ( $y$ direction) magneticfield distribution in the laser system $A$ and $C$, respectively. As the LDW laser operates at the band edge, an oscillation is built up and strongly localized in the LDW area. The electromagnetic-field outside the structure in system $A$ is apparently weaker than that in system $C$. The LDW laser output depends on the butt joint waveguide structure which is investigated theoretically and experimentally. For system $A$, $B$, and $C$, the maximum output power at pulse peak was $0.047,7.3$, and $10 \mu \mathrm{W}$, respectively, corresponding to the pump peak power of $139 \mu \mathrm{W}$ in the experiment. The output power of system $C$ was 214 times higher than that of $A$.

The output power is proportional to $1 / Q_{i}, i$ for $A, B$, and $C$ respectively. The output power ratio of device $C$ to $A$ could be numerically estimated. By FDTD simulation, the $Q$ factors for the three waveguide devices represented by $Q_{A}$, $Q_{B}$, and $Q_{C}$ are of 119000,2160 , and 1411, respectively, for the LDW model with 38 period lengths, which is 34 periods less than the real structure. From this numerical estimation, the output power ratio of $C$ to $A$ is only 85 , which is much less than the experimental result. However, the output ratio of $C$ to $B$ is 1.53 , which is consistent with the experimental result of 1.37 quite well. The LDW laser possesses higher $Q$ factor with the increase in lattice periods. Considering the simulation model with only 37 periods is much smaller than real structure of 85 periods shown in Fig. 1, a model with 55 periods waveguide is simulated limited by the calculation capacity of the computer, and a higher $Q_{A}$ of 332800 is derived. However for devices $B$ and $C$, the added periods work only as photon propagating waveguide, no matter how long the coupled waveguide is, it just devotes to propagate the photons. It does not work as part of the cavity, so the $Q$ factor for devices $C$ and $B$ does not change when the output coupler is lengthened. For device $A$, the added structures, here we changed the model of 37 to 55 periods, work not only as an output coupler, but also as a part of the cavity for the resonant mode standing at the band edge in the output waveguide. Therefore the more periods it has, the higher the $Q$ factor will be especially for device $A$. From this simulation result, we deduce that the output ratio of $C$ to $A$ is $\sim 238$, which agrees with the experimental result.

For the experimental result, we first considered that zero group velocity of waveguide $A$ disturbed the efficient output because the output waveguide worked as a part of the cavity. In another experiment, however, we also observed a comparable low output power for the various point defect lasers, each of which was evanescent coupled to the waveguide through a PC mirror. Therefore, we should rather evaluate the effectiveness of device $C$ structure. It could be attributed to the efficient light transmission of the zero group velocity light to the waveguide through the chirped structure output coupler. For the maximum output power for device $C$, the external differential quantum efficiency is estimated to be $20 \%$. This value is remarkable when considering the absorption loss of unexcited waveguide region.

In conclusion, we obtained a marked enhancement of light output from a PC micron laser by employing the butt joint of line defect laser and output waveguide with the chirped structure. The 214 times output enhancement in experiment is less than the theoretical prediction of 238 . We address such difference to the disturbance of the uniformity of the PC structure due to the immatured large area microfabrication. The external differential quantum efficiency is estimated to be $20 \%$, which is a practical level recorded for PC lasers. We believe that this is a promising result for largescale active photonic integration in the PCs.

This work was supported by the Chinese National Key Basic Research Special Fund (CNKBRSF) (Grant No. 2006CB921700), the National High-Technology Research and Development Program of China (Grant No. 2006AA03Z403), and the National Natural Science Foundation of China (NNSFC) (Grant Nos. 10634080 and 60677046).

${ }^{1}$ A. Sugitatsu and S. Noda, Electron. Lett. 39, 213 (2003).

${ }^{2}$ A. Sugitatsu, T. Asano, and S. Noda, Appl. Phys. Lett. 84, 5395 (2004).

${ }^{3}$ A. Sugitatsu, T. Asano, and S. Noda, Appl. Phys. Lett. 86, 171106 (2005).

${ }^{4}$ S. Noda, A. Chutinan, and M. Imada, Nature (London) 407, 608 (2000).

${ }^{5}$ K. Nozaki, H. Watanabe, and T. Baba, Appl. Phys. Lett. 92, 021108 (2008).

${ }^{6}$ A. Faraon, E. Waks, D. Englund, I. Fushman, and J. Vuckovic, Appl. Phys. Lett. 90, 073102 (2007).

${ }^{7}$ E. Waks and J. Vukovic, Opt. Express 13, 5064 (2005)

${ }^{8}$ C. Seassal, Y. Desieres, X. Letartre, C. Grillet, P. Rojo-Romeo, P. Viktorovitch, and T. Benyattou, IEEE J. Quantum Electron. 38, 811 (2002).

${ }^{9}$ G. H. Kim, Y. H. Lee, A. Shinya, and M. Notomi, Opt. Express 12, 6624 (2004).

${ }^{10}$ P. T. Lee, T. W. Lu, C. M. Yu, and C. C. Tseng, Opt. Express 15, 9450 (2007).

${ }^{11}$ B. Song, S. Noda, T. Asano, and Y. Akahane, Nat. Mater. 4, 207 (2005).

${ }^{12}$ W. H. Zheng, G. Ren, X. T. Ma, X. H. Cai, L. H. Chen, K. Nozaki, and T. Baba, J. Cryst. Growth 292, 341 (2006). 